\title{
A Critical Look at 2015 Acute Flaccid Paralysis (AFP) Surveillance Core Indicators of Bauchi State, Nigeria
}

\author{
Jalal-Eddeen Abubakar Saleh", , Rui Gama Vaz², Fiona Braka ${ }^{3}$, Khaled Abdelrahim¹, \\ Adamu Ibrahim Ningi ${ }^{1}$
}

${ }^{1}$ EPI-IVE, World Health Organization, Bauchi Zonal Office, Bauchi State, Nigeria

${ }^{2}$ WR/NIE, World Health Organization, Abuja, Nigeria

${ }^{3}$ EPI-IVE, World Health Organization, Abuja, Nigeria

Email address:

drjalals@yahoo.com (Jalal-Eddeen A. S.)

${ }^{*}$ Corresponding author

\section{To cite this article:}

Jalal-Eddeen Abubakar Saleh, Rui Gama Vaz, Fiona Braka, Khaled Abdelrahim, Adamu Ibrahim Ningi. A Critical Look at 2015 Acute Flaccid Paralysis (AFP) Surveillance Core Indicators of Bauchi State, Nigeria. Science Journal of Public Health.

Vol. 4, No. 4, 2016, pp. 326-329. doi: 10.11648/j.sjph.20160404.19

Received: May 8, 2016; Accepted: May 17, 2016; Published: July 4, 2016

\begin{abstract}
Background: Poliomyelitis, a disease that predominantly affects children under the age of five years, is a highly infectious viral disease transmitted through the faeco-oral route. The disease could result in irreversible paralysis in 1:200 infections and has a case fatality rate of $5-10 \%$ especially when the respiratory muscles become immobilized. The Global Polio Eradication Initiatives (GPEI) is a partnership between international organizations and governments of countries and other stakeholders aimed at polio eradication across the globe through the use of strategies such as improved AFP surveillance, strengthening routine immunizations, and supplemental immunization activities. Importantly, the use of polio vaccine in multiple times on a child confers lifelong immunity against the disease. Study Design: Retrospective study Methods: Quantitative crosssectional study of AFP cases reported across the 20 local government areas (LGA) of Bauchi State, northeastern Nigeria. The data was collected between $1^{\text {st }}$ January 2015 and $31^{\text {st }}$ December 2015. Results: Although all the 20(100\%) LGAs met the non-polio AFP rate (NPAFP) of reporting at least 1 case per annum and stool adequacy, only 10(50\%) LGAs met the minimum accepted NPENT rate of $10 \%$ with the remaining $10(50 \%)$ LGAs falling short of the minimum acceptable rate. Thus, the cumulative NPENT rate for Bauchi State stood at 10.3\%. Conclusion: The 2015 AFP surveillance results from Bauchi State clearly support the remarkable progress made by Nigeria. However, there is need for future studies to establish reasons behind the remaining 10 $(50 \%)$ of the LGAs in Bauchi failing short of meeting the minimum NPENT rate of 10.
\end{abstract}

Keywords: Acute Flaccid Paralysis, Surveillance, Non-polio Enterovirus, Non-polio-AFP Rate, Eradication, Poliomyelitis, Stool Adequacy

\section{Introduction}

Poliomyelitis (Polio) is a highly infectious viral disease transmitted through faeco-oral route and mainly affects children under the age five years. On entry into the human system, the virus multiplies in the intestine of its host and subsequently invades the nervous system, which may result in total paralysis within couple of hours. It has been shown that 1 in 200 infections could result in irreversible paralysis with case fatality rate of $5-10 \%$ when the respiratory muscles become immobilized. Although there is no cure for polio infection, use of polio vaccine multiple times on a child confers lifelong immunity against the disease $[1,2]$.

The 1988 at the forty-first World Health Assembly gave birth to the Global Polio Eradication Initiative (GPEI). The programme launch was spearheaded by national governments, the World Health Organization (WHO), the US Centers for Disease Control and Prevention (CDC), the UNICEF, and Rotary International. This effort was strongly supported by key partners especially the Bill and Melinda Gates Foundation (BMGF). The effort of global key players to protect children from polio disease from across the globe has resulted in the 
reduction in number of cases with at least 99\% [1-3].

The international accepted gold standard for detecting poliomyelitis cases is through AFP (acute flaccid paralysis) surveillance. The AFP surveillance system essentially has four steps: active case search and reporting of children with suspected AFP, collection and transportation of stool samples for laboratory analysis, laboratory isolation and identification of poliovirus, and mapping of the virus to determine the its origin [1-3].

The global effort towards polio eradication, success of which depends on adequate funding, has expanded additional capacities to tackle other infectious diseases through improved surveillance and strengthening of the immunization system. It is a result of this effort that the global number of polio cases has decreased by over $99 \%$ from an estimated 350,000 to 359 reported cases from 1988 to 2014 [1, 2].

Importantly, record shows that the WHO region of the Americas was in 1994 certified as polio-free. This historic milestone opened up doors for countries and regions to get polio-free certifications from the WHO. Subsequent poliofree certifications were the WHO Western Pacific region in 2000, the WHO European region in 2002, and the WHO South-East Asia region in 2014. Thus, the global eradication initiative was able to ensure that globally, there are at least $80 \%$ living in certified polio-free regions [1].

Nigeria, prior getting delisted in July 2015, was among the three polio-endemic countries of the world. It is encouraging to note that there are only 2 remaining polio-endemic countries (Afghanistan and Pakistan) down from more than 125 in 1988. Looking at the global polio statistics, in 2015, there are 70 cases of WPV all from endemic countries with $26 \mathrm{cVDPV}$ (3 from endemic and 23 from non-endemic countries). In Nigeria, 2015 was a historic year as it got delisted from the list of polio endemic countries with a record of only a single case of cVDPV with no any case of wild poliovirus (WPV). In comparison with 2014, the country had 28 cases of cVDPV with 6 WPV. The last WPV1 and WPV3 recorded in Nigeria had their date of onset of paralysis as $24^{\text {th }}$ July 2014 and $10^{\text {th }}$ November 2012 respectively [1]. This encouraging result clearly shows that all things being equal, the country is expected to get certification by the WHO in 2017.

Bauchi State is agrarian in nature and has an estimated population of 4,653,066 [4]. With a total landmass of 49,119 $\mathrm{km}^{2}$, the State is geographically located in the northeastern part of Nigeria between latitudes $9^{\circ} 3^{\prime}$ and $12^{\circ} 3^{\prime}$ north of the equator, and longitudinally $8^{\circ} 50^{\prime}$ and $11^{\circ}$ east of the Greenwich meridian. The 2015 population for children under the age of 15 years stands at 3,007,527. Bauchi State shares national borders with Gombe, Taraba, and Yobe to the East, Jigawa and Kano to the North, Kaduna to the West, and Plateau to the South. Bauchi has cultural and religious similarities with these neighboring States thus making it a transit point especially for trans-border activities [5].

Globally, the acceptable gold standard for detecting cases of poliomyelitis is through AFP (acute flaccid paralysis) surveillance and the steps are essentially four; active case search and reporting of children with suspected AFP, collection and transporting of stool samples for laboratory analysis, laboratory isolation and identification of poliovirus, and mapping of the virus to determine the its origin. Emphasis is on countries to meet the two most important AFP core indicators: non-polio AFP rate (NP-AFP rate) and the stool adequacy rates [1-3].

The NP-AFP rate, which tests sensitivity of the AFP surveillance system, stipulates that there should be at least one detected case of non-polio AFP annually per 100000 population of children under the age of 15 years [1-3].

The stool adequacy rates require that at least $80 \%$ of suspected AFP cases should have adequate stool specimens collected and analyzed. For stool to be adequate, the specimen must be two stools samples of sufficient quantity of at least 8 grams for laboratory analysis, collected between 24-48 hours, within 14 days of the onset of paralysis, and to well documented and must arrive the laboratory by reverse cold chain. Additionally, the specimen must reach the laboratory within 72 hours of collection of the second sample. Anything short of these would render the stool specimen as inadequate also affecting the non-polio enteroviruses (NPENT) in the specimen thus comprising the outcome of the stool analysis. Another important indicator often used to gauge sensitivity of the AFP surveillance system is the NPENT rate, which is fixed at $\geq 10 \%[1-3]$.

\section{Methods}

This retrospective cross-sectional quantitative research used the number of AFPs reported across the 20 LGAs in Bauchi State, Nigeria and the 2015 laboratory results available as at $30^{\text {th }}$ March 2016. The AFPs reported were between $1^{\text {st }}$ January $-31^{\text {st }}$ December 2015 by WHO trained disease surveillance and notification officers (DSNOs) through identified key informants and subsequently verified by WHO surveillance officers using a standard AFP case investigation form. Informed of consent was obtained from the local authorities and or parents of these children prior to administering the questionnaire. The participants were well informed in the local language that they understood for the purpose of administering questionnaires. The information obtained was securely kept in the official WHO database, and restricting data access except to authorize persons. The data, archived at the WHO State office, was accessed for this analysis after an approval was obtained from the State Coordinator. The data available as at $30^{\text {th }}$ March 2016 shows that 534 AFPs were reported and duly verified from across the 20 LGAs in 2015.

The various columns on the AFP case investigation form (CIF) include: column for identification/demographic information, clinical history, vaccination history, and stool specimen collection, and how it was transported to the laboratory for final analysis. The quality of the items on the CIF was sought with content validity, and the outcome is valid and generalizable. Thus, the instrument clearly measures what it was expected to measure as validity and reliability of the measurement instrument was well tested $[6,7]$. 


\section{Results}

Table 1. Reported AFPs across the 20 LGAs in Bauchi State in 2015.

\begin{tabular}{lll}
\hline LGA & Reported AFPs & Percentage (\%) \\
\hline Alkaleri & 23 & $4.31 \%$ \\
Bauchi & 50 & $9.36 \%$ \\
Bogoro & 17 & $3.18 \%$ \\
Dambam & 41 & $7.68 \%$ \\
Darazo & 36 & $6.74 \%$ \\
Dass & 18 & $3.37 \%$ \\
Gamawa & 32 & $5.99 \%$ \\
Ganjuwa & 15 & $2.81 \%$ \\
Giade & 26 & $4.87 \%$ \\
Itas/Gadau & 24 & $4.49 \%$ \\
Jama'are & 37 & $6.93 \%$ \\
Katagum & 28 & $5.24 \%$ \\
Kirfi & 21 & $3.93 \%$ \\
Misau & 22 & $4.12 \%$ \\
Ningi & 11 & $2.06 \%$ \\
Shira & 16 & $3.00 \%$ \\
Tafawa-Balewa & 30 & $5.62 \%$ \\
Toro & 50 & $9.36 \%$ \\
Warji & 11 & $2.06 \%$ \\
Zaki & 26 & $4.87 \%$ \\
Grand Total & 534 & $100.00 \%$ \\
\hline
\end{tabular}

This study uses 2015 AFP results available from the national polio reference laboratory. Based on the results, there are 534 reported AFP cases from across the 20 LGAs of Bauchi State between $1^{\text {st }}$ January 2015 and $31^{\text {st }}$ December 2015. However, there are other results that are still pending and yet to be released by the national polio reference laboratory.

Table 1 presents the frequency of AFPs reported and verified by each of the LGAs in 2015. Although all the 20 LGAs met the minimum required annual NPAFP rate, Bauchi $50(9.36 \%)$ and Toro 50(9.36\%) LGAs reported the highest number of cases in the State in 2015. However, the LGAs that reported the lowest number of AFPs were Ningi $11(2.06 \%)$ and Warji 11(2.06\%).

Table 2 is a comparison of AFPs reported in 2015 (534) with that of 2014 (371). It could be seen that in 2015, there was an increase of 163 AFP cases. Still, looking at the cases in 2014, Bauchi $34(9.16 \%)$ and Toro 33(8.89\%) LGAs reported the highest number of AFP cases as compared to other LGAs. This remarkable difference is also shown in a pictorial form in Figure 1.

Table 2. Reported AFPs across the 20 LGAs in Bauchi State in 2015 compared with 2014.

\begin{tabular}{lll}
\hline LGA & Reported AFPs IN 2015 & Reported AFPs IN 2014 \\
\hline Alkaleri & $23(4.31 \%)$ & $21(5.66 \%)$ \\
Bauchi & $50(9.36 \%)$ & $34(9.16 \%)$ \\
Bogoro & $17(3.18)$ & $12(3.23 \%)$ \\
Dambam & $41(7.68 \%)$ & $31(8.36 \%)$ \\
Darazo & $36(6.74 \%)$ & $25(6.74 \%)$ \\
Dass & $18(3.37 \%)$ & $15(4.04 \%)$ \\
Gamawa & $32(5.99 \%)$ & $14(3.77 \%)$ \\
Ganjuwa & $15(2.81)$ & $10(2.70 \%)$ \\
Giade & $26(4.87 \%)$ & $10(2.70 \%)$ \\
Itas/Gadau & $24(4.49 \%)$ & $10(2.70 \%)$ \\
Jama'are & $37(6.93 \%)$ & $31(8.36 \%)$ \\
Katagum & $28(5.24 \%)$ & $20(5.39 \%)$ \\
\hline
\end{tabular}

\begin{tabular}{lll}
\hline LGA & Reported AFPs IN 2015 & Reported AFPs IN 2014 \\
\hline Kirfi & $21(3.93 \%)$ & $20(5.39 \%)$ \\
Misau & $22(4.12 \%)$ & $16(4.31 \%)$ \\
Ningi & $11(2.06 \%)$ & $10(2.70 \%)$ \\
Shira & $16(3.00 \%)$ & $9(2.43 \%)$ \\
Tafawa-Balewa & $30(5.62 \%)$ & $21(5.66 \%)$ \\
Toro & $50(9.36 \%)$ & $33(8.89 \%)$ \\
Warji & $11(2.06 \%)$ & $9(2.43 \%)$ \\
Zaki & $26(4.87 \%)$ & $20(5.39 \%)$ \\
Grand Total & 534 & 371 \\
\hline
\end{tabular}

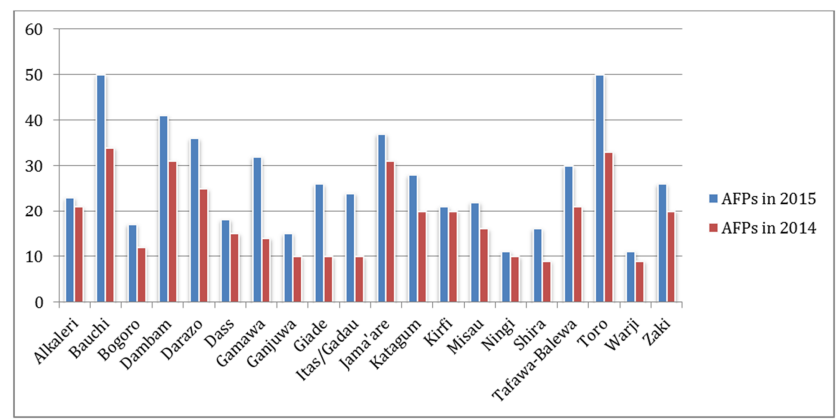

Figure 1. Chart showing reported AFPs across the 20 LGAs in Bauchi State in 2015 as compared with 2014.

Table 3. Showing NPENT rate of AFPs reported in Bauchi State in 2015.

\begin{tabular}{lll}
\hline LGA & No. of AFP & NPENT rate \\
\hline Alkaleri & 23 & 0 \\
Bauchi & 50 & 6.1 \\
Bogoro & 17 & 8.3 \\
Dambam & 41 & 2.9 \\
Darazo & 36 & 11.5 \\
Dass & 18 & 6.7 \\
Gamawa & 32 & 13.3 \\
Ganjuwa & 15 & 30 \\
Giade & 26 & 38.5 \\
Itas/Gadau & 24 & 30 \\
Jama'are & 37 & 5.7 \\
Katagum & 28 & 10 \\
Kirfi & 21 & 10 \\
Misau & 22 & 11.8 \\
Ningi & 11 & 0 \\
Shira & 16 & 0 \\
Tafawa-Balewa & 30 & 19 \\
Toro & 50 & 8.6 \\
Warji & 11 & 22.2 \\
Zaki & 26 & 9.5 \\
Grand Total & 534 & 10.3 \\
\hline
\end{tabular}

Table 3 shows the NPENT rate of the 20 LGAs across the State. Although Bauchi State narrowly managed to achieve a cumulative NPENT rate of 10.3, it is not encouraging to note that there are only $10(50 \%)$ out of the 20 LGAs that achieved the minimum required NPENT rate of 10. Thus, the two LGAs that reported the highest number of AFPs in 2015 (Bauchi and Toro) had low NPENT rates of 6.1 and 8.6 respectively, falling short of the required minimum NPENT rate of 10. However, this low NPENT rate poses a question on the quality of stool specimen sent to the laboratory for analysis.

In Figure 2, which shows stool adequacy of the 20 LGAs across the State as reported in 2015, all but two LGAs achieved $100 \%$ stool adequacy rate, which is far above the 
minimum required level of $80 \%$. The two LGAs with stool adequacy of $96 \%$ and $97 \%$ respectively are Alkaleri and Gamawa LGAs.

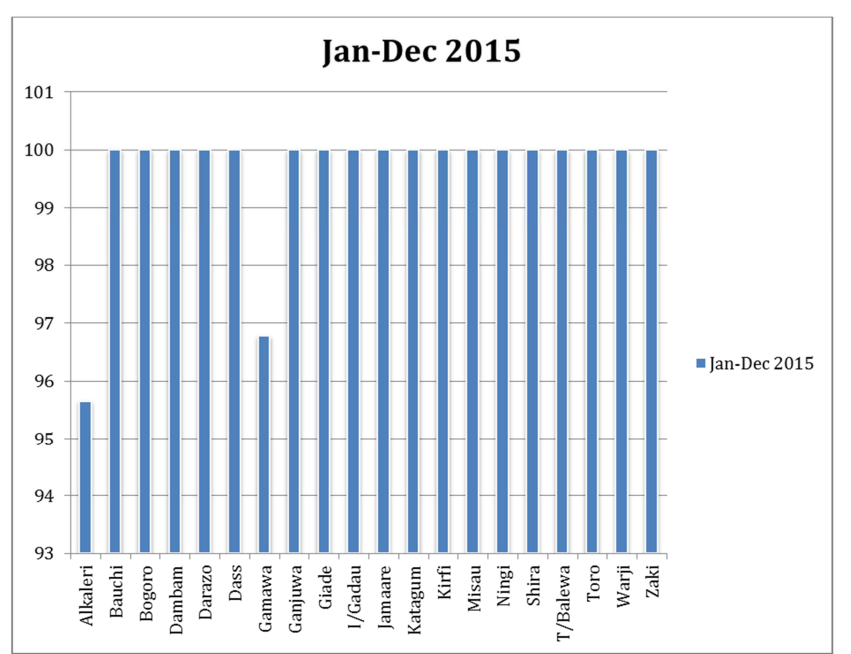

Figure 2. Chart showing stool adequacy rate of AFPs reported across the 20 LGAs in Bauchi State in 2015.

\section{Discussions}

Although Bauchi State is rated as one of the very high risk LGAs in the on-going PEI in Nigeria, findings from this study indicates that in 2015, the State met the AFP core indicators. However, there are still unanswered questions as to reasons behind the low NPENT rate recorded by 10 LGAs in 2015 hence the need to probe further and establish the possible factors associated with the low non-polio enteroviruses (NPENT) rate in 2015 so as to avoid future occurrence especially now that Nigeria is billed to get certification as polio free country in 2017.

Importantly, the NPENT group of viruses that include Coxsackie virus A, Coxsackie virus B, Echoviruses, Enterovirus D68 and other Enteroviruses. The viruses annually cause millions of infections globally resulting in the hospitalization of affected persons especially infants, children, and teenagers under the age of fifteen years. While, most of those who get infected with the NPENT viruses do not fall sick, infants and other individuals with poor immunity are liable to have complications that may present with weakness of any of the limbs that reverses after a given period of time from the date of onset. Suspected AFP cases, which often present with weakness or inability to use one or more of the four limbs, are caused either by the enteroviruses, (poliovirus or NPENT viruses). Thus, stools specimen from suspected non-polio AFP case sent to national polio laboratories should test positive for the enteroviruses to achieve the NPENT rate $[8,9]$.

\section{Acknowledgments}

I acknowledge, with gratitude, approval for the use of the AFP dataset from the WHO office Bauchi for the sole purpose of this study.

\section{Conflicts of Interest}

There is no conflict of interest or royalty associated with this study.

\section{References}

[1] WHO (2015). Poliomyelitis. Retrieved from http://www.who.int/mediacentre/factsheets/fs114/en/

[2] Global Polio Eradication Initiative (2016). Retrieved from http://www.polioeradication.org/

[3] CDC (2015). The Global Polio Eradication Initiative Stop Transmission of Polio (STOP) Program - 1999-2013. Retrieved from http://www.cdc.gov/mmwr/preview/mmwrhtml/mm6224a5.ht $\mathrm{m}$

[4] National Population Commission of Nigeria (2006). Retrieved from www.population.gov.ng/ -Cached

[5] Nigerian National News (2012): Bauchi Retrieved from $\mathrm{http}: / /$ nnn.com.ng/?page_id $=452$

[6] Frankfort-Nachmias, C., \& Nachmias, D. (2008). Research methods in the social sciences (7th ed.). New York: Worth.

[7] Szklo, M., \& Nieto, F. J. (2014). Epidemiology: Beyond the basics (3rd ed.). Sudbury, MA: Jones and Bartlett

[8] CDC (2015). Non-Polio Enterovirus. Retrieved from http://www.cdc.gov/non-polio-enterovirus/

[9] CDC (2013). Non-Polio Enterovirus. Retrieved from http://www.cdc.gov/non-polio-enterovirus/about/ 